\title{
Conventional oral and secondary high dose vaginal metronidazole therapy for recurrent bacterial vaginosis: clinical outcomes, impacts of sex and menses
}

This article was published in the following Dove Press journal:

Infection and Drug Resistance

Jack D Sobel'

Navkiranjot Kaur ${ }^{2}$

Nicole A Woznicki'

Dina Boikov'

Tina Aguin ${ }^{3}$

Gurveer Gill ${ }^{2}$

Robert A Akins ${ }^{2}$

'Division of Infectious Diseases, Wayne State University School of Medicine, Detroit, MI, USA; ${ }^{2}$ Department of Biochemistry, Microbiology, and Immunology, Wayne State University School of Medicine, Detroit MI, USA; ${ }^{3}$ Department of Obstetrics and Gynecology, Wayne State University School of Medicine, Detroit, MI, USA

Correspondence: Robert A Akins Department Biochemistry, Microbiology, and Immunology, Wayne State University School of Medicine, 540 East Canfield Dr. Detroit, MI 4820I, USA

Tel + I 3135770419

Fax +I313577 II55

Email rakins@med.wayne.edu
Purpose: Oral metronidazole therapy is the standard of care for bacterial vaginosis (BV), yet it has alarming rates of recurrence and refractory responses among recurrent BV (RBV) patients. This study addresses whether high dose vaginal metronidazole therapy (HDM) is beneficial in RBV patients who fail after standard of care (SOC) therapy, whether diagnostic test scores proximal to the HDM predict clinical outcome, and whether menses, coitus, or race influences therapy outcome.

Patients and methods: A total of 90 patients with RBV were given SOC and tracked 74 for up to 9 months. Refractory or recurrent patients (57) with symptomatic BV were given HDM and followed for up to 8 months. Patients were evaluated by Amsel criteria, Nugent score, and a qPCR assay that assesses the Lactobacillus content.

Results: HDM achieved at least short-term remission in $68 \%$ of the patients who were refractory to or recurred after SOC and provided a 10-day increase in the mean duration of remission among patients who eventually recurred $(p=0.027)$. Patients with prolonged dysbiosis ( $\mathrm{pH}>5$ or Amsel 4) before symptomatic recurrence were more likely to recur after subsequent HDM. Most recurrence happened within 10 days of menses, but sex in this cohort was not associated with clinical outcome. Mean diagnostic BV scores of African American patients in remission were inferior to scores of a small cohort of Caucasian patients in remission.

Conclusion: Encouraging results obtained with HDM justify a prospective, randomized study to determine if follow-up HDM is beneficial among a broader cohort of women failing conventional oral metronidazole therapy.

Keywords: nugent score, amsel criteria, LbRC, prognosis of bacterial vaginosis

\section{Introduction}

Oral divided dose metronidazole is the most widely used therapy and serves as the standard of care for bacterial vaginosis. Despite its favorable pharmacodynamics, recurrence rates of $\mathrm{BV}$ are high, $69-80 \%$ within 12 months. ${ }^{1,2}$ The causes of recurrence are still not known, whether from resistant subpopulations of pathogenic bacteria, ${ }^{3-6}$ reinfection, ${ }^{7-11}$ or host factors. ${ }^{12}$ Poor efficacies have incentivized use of alternative treatments, including higher and more prolonged nitroimidazole dosing, ${ }^{13}$ achieving higher concentrations with local vaginal gels, ${ }^{13-17}$ or use of or supplementation with other agents such as boric acid, lactic acid, vitamin C, lactoferrin, subtilosin, lauramide arginine ethyl ester, plant extracts from Myrtus Communis and Berberis Vulgaris, ${ }^{13,18-26}$ prebiotics, ${ }^{27,28}$ and probiotics, detailed in our Discussion. Prolonged maintenance therapy with vaginal 
metronidazole temporarily reduces recurrence in otherwise highly recurrent patients, but patients frequently relapse after this therapy is discontinued. ${ }^{16}$ Some species, notably $G$. vaginalis and $A$. vaginae remain prevalent even after prolonged periodic presumptive treatment with $\mathrm{HDM}^{29}$ We recently validated that standard of care (SOC) oral metronidazole has poor efficacy among recurrent, largely African American patients, showing alarming $76 \%$ refractory or recurrent responses. ${ }^{30}$

In the present study, we treated RBV patients who failed after oral metronidazole with high dose vaginal metronidazole. We assess benefits of this regimen and whether outcome is associated with menses or race and if outcome can be predicted from BV diagnostic test scores.

\section{Materials and methods}

\section{Enrollment and therapies}

This single cross-over prospective pilot study was performed at a Vaginitis Clinic at Wayne State University in Detroit, MI, and enrolled RBV patients from September 30, 2014 to December 1, 2017. RBV patients were defined as those diagnosed with BV 3 or more times in the year prior to enrollment. A total of $90 \mathrm{RBV}$ patients were given SOC oral metronidazole therapy. Enrollment strategies, exclusion criteria, and other clinical parameters and definitions are detailed in the first arm of this study that focused on response to oral metronidazole therapy. ${ }^{30}$ Major requirements for enrollment as recurrent BV patients were: a history of $\geq 3$ episodes of symptomatic BV in the previous year, positive at enrollment for $\geq 3$ Amsel criteria (vaginal $\mathrm{pH} \geq 4.5$, positive amine "Whiff" test, $>20 \%$ clue cells, and grayish-white adherent discharge), ${ }^{31}$ symptomatic (odor, discharge, discomfort, or itching), premenopausal, $\geq 18$ years old, heterosexual, no mixed vaginal infections, willing to refrain from using any other vaginal products during the study period, willing to either use condoms for the duration of the study or to report unprotected sex, willing to abstain from coitus within 48 hrs of any study visit, and willing to abstain from alcohol during therapy. Symptomatic patients with Amselconfirmed BV were prescribed oral metronidazole $500 \mathrm{mg}$ bid for 7 days and seen on average 16 days following initiating therapy and monthly for up to 9 months. Patients returning after SOC therapy with symptoms of BV and $\geq 3$ Amsel criteria were considered recurrent or refractory if remission was never achieved and were given high dose metronidazole (HDM): $750 \mathrm{mg}$ metronidazole per $200 \mathrm{mg}$ miconazole vaginal suppositories (Embil Pharmaceutical Co. Ltd, Istanbul, Turkey), once daily for 7 days, and followed monthly as described. Patients were followed for up to 8 months unless they acquired other exclusion criteria or because of a recurrence after HDM. Ethically, we could not repeat identical treatments that were failing in the latter group. Accordingly, outside of this study, they were offered a maintenance antimicrobial regimen previously reported. ${ }^{16}$ All patients also performed daily vaginal self-swabs following provided instructions and recorded whether they engaged in sex, with or without a condom, and when they experienced menses. The protocol was approved by Wayne State University Institutional Review Board (IRB 040314M1F) and enrolled patients with written informed consent in compliance with the Declaration of Helsinki; the full protocol is posted online at https://docs.google.com/document/ d/1uUZvo0soD2rBCDtMBxsT1TmAJp72CD2E E6FoWM9Ato/edit?usp=sharing.

RBV following study treatment is defined as bacteriological or therapeutic recurrence following at least one posttreatment visit of remission. Bacteriological recurrence is a Nugent score $\geq 7$; therapeutic recurrence is defined as Amsel $\geq 3$ and symptoms presented by the patient requiring treatment. Remission was defined as the absence of recurrence; long-term remission is the absence of recurrence for at least 3 months or to the end of the study. Refractory patients were defined as women with persistent symptoms and Amsel scores 3-4 at the first follow-up visit occurring within 30 days of initiating therapy.

\section{DNA extraction and qPCR testing}

DNA was extracted from freshly obtained vaginal swabs or swabs suspended in $4 \mathrm{~mL}$ of 2 -isopropanol as described. ${ }^{30}$ The relative composition of Lactobacillus in these DNA preparations was assessed by the qPCR assay called LbRC. ${ }^{30}$ This assay amplifies each DNA twice with a broad-spectrum bacterial 16S ribosomal RNA primer set which flanks the V3-V4 variable domains; the second amplification includes nonextendable oligomers that bind specifically to all vaginal Lactobacillus species and, by partially overlapping or juxtaposing the broad-spectrum primers, block their amplification. The LbRC/5 test does not differentiate between $L$. iners and the other Lactobacillus species. The comparison of the cycles of threshold quantification $(\mathrm{Cq})$ values generates a $\Delta \mathrm{Cq}$; a large value indicates Lactobacillus dominance. This value adds penalties to the score if melting curve peaks show Tm values other than Lactobacillus, comprise the LbRC score. qPCR assays were performed as described, ${ }^{30}$ using a threshold of $\leq 1$ to define $\mathrm{BV}$, and $\leq 5$ as a prognostic indicator of later recurrence. 


\section{Statistical methods}

Statistical significance and other analyses followed the STARD guidelines ${ }^{32}$ as cited in the text and were performed using GraphPad Prism version 6.00 for Windows, GraphPad Software, La Jolla California, USA. Categorical data for diagnostic accuracy were analyzed via Chi-square or Fisher's exact test to generate 95\% confidence intervals. Individual results being analyzed for pair-wise comparisons between assays in which one assay result was missing were excluded. Significance of comparisons of continuous data was analyzed by unpaired $t$-tests if the distributions passed the D'Agostino-Pearson omnibus normality test or by the Mann-Whitney test if not. ANOVA tests with multiple comparisons were performed with post hoc testing as indicated. $P \leq 0.05$ was considered significant.

\section{Results}

\section{Short-term efficacies of SOC versus HDM}

As reported previously, ${ }^{30} 74$ of 90 women enrolled who received SOC therapy were tracked at follow-up visits (Figure 1). Seventeen of 74 tracked patients (24\%) achieved long-term remission with eradication of $\mathrm{BV}$ for at least 3 months of follow-up, with a mean tracked duration of 194 \pm 84 days. Noteworthy, 29 patients (39\%) failed to respond and were refractory to SOC therapy as evaluated at the first follow-up visit. The final group of 28 additional patients $(38 \%)$ responded well in the short term only, then recurred with symptomatic BV, with a mean interval of $62 \pm 43$ days. Among the 57 women, either refractory or recurrent, who received HDM vaginal therapy, 38 were available for longterm follow-up after HDM therapy (Figure 1).

Following HDM, 8 of the 38 tracked patients (21\%) achieved long-term remission, mean 204 \pm 85 days, not significantly different than the duration of remission after SOC (paired $t$-test $p=0.772$ ). However, the SOC refractory versus recurrence groups showed very different responses to HDM. Among SOC refractory patients followed $(n=21), 10$ were similarly refractory to HDM therapy (48\%), although 3 patients (14\%) did achieve longterm remission and a further 8 patients (38\%) initially responded but suffered early recurrence. These results indicate that women refractory to SOC have high likelihood of being refractory to HDM therapy. In contrast, among the cohort of women initially responding to SOC only to experience an early recurrence, refractory

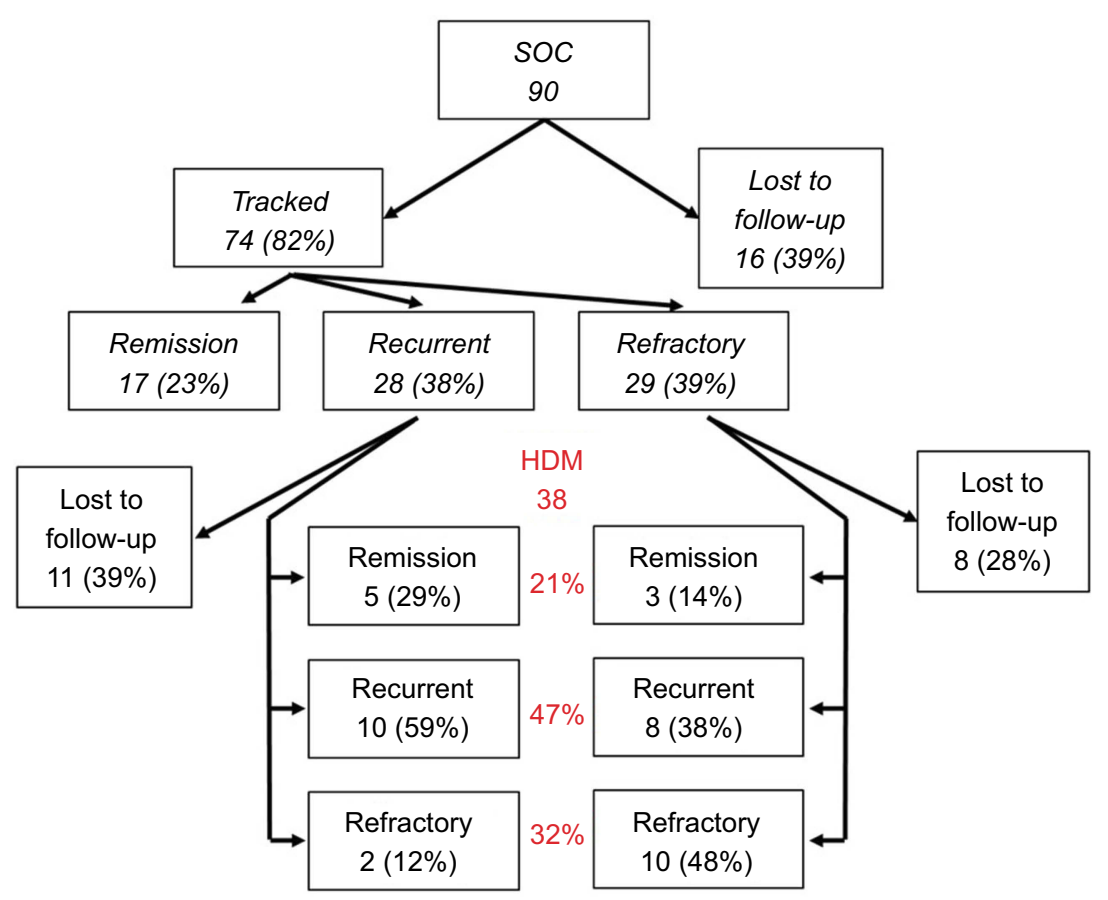

Figure I Flow diagram of enrollment and responses to therapy. SOC oral metronidazole therapy results (italics) were reported previously. Copyright @American Society for Microbiology, J. Clin, Microbiol. 57(5):pii:e00227-19. doi:10.128/JCM.0227-19. Remission patients were those who remained in clinical remission (Amsel 0-2, asymptomatic) for at least 3 months. Recurrent patients were those that achieved clinical remission for at least one month before recurring, defined here as Amsel $3-4$ with symptoms warranting treatment. Refractory patients were those who did not achieve clinical remission after therapy. Percentages were calculated based on the subtotals in the upstream boxes. Percentages in red are calculated from the entire tracked HDM cohort.

Abbreviations: SOC, standard of care; HDM, high dose vaginal metronidazole therapy. 
responses to HDM were infrequent (2 of 16, 12\%). An additional 5 patients (29\%) achieved long-term admission. However, more than half (10 of 17 or $59 \%$ ) experienced a further or second recurrent episode following HDM therapy. The reduced refractory responses following SOC-recurrence versus following SOC-refractory responses were significant (Fisher exact $p=0.034$ ). Despite the recurrent and refractory responses, 26 of 38 patients $(68 \%)$ achieved at least short-term remission by the follow-up HDM therapy.

\section{Comparisons of BV test scores before and after SOC versus HDM therapy}

One explanation for at least short-term remission after HDM follow-up treatment of patients who recurred after or were refractory to SOC is that these episodes were not as severe or dysbiotic as the initial episode that confronted SOC therapy. In particular, perhaps the subset of SOC failures by recurrence was less severe and therefore these patients had significantly fewer refractory responses $(12 \%)$ after HDM therapy than the SOC refractory group (48\%). If so, comparisons of BV diagnostic test scores among these subgroups at the time treatments were prescribed should be significantly different.

BV test score distributions were very similar at the two symptomatic BV visits, ie, the initial visit at which SOC therapy was prescribed and the recurrence/refractory visit, treated with HDM. Mean $\mathrm{pH}$ was incrementally but significantly higher at the SOC visit, $5.7 \pm 0.6$ versus $5.4 \pm 0.7$ $(p=0.009)$. Mean Amsel scores were also slightly higher at the SOC visit, $3.9 \pm 0.4$, than at the $\mathrm{HDM}$ visit, $3.5 \pm 0.1$ $(p=0.001)$. Of note, however, differences in mean Nugent and $\mathrm{LbRC} / 5$ scores were not significant (Mann-Whitney tests, $p>0.192$ ).

Similarly, mean BV test scores at the post-SOC visit were similar to the post-HDM visit scores. Differences in mean $\mathrm{pH}$ after SOC versus after HDM were incrementally but significantly lower $(4.8 \pm 0.7$ versus 5.2 \pm 0.8 ). Differences in mean Amsel, Nugent, LbRC/5 and $\Delta \mathrm{Cq}$ scores were insignificant (Mann-Whitney tests, $p>0.547$ ).

Finally, comparisons of BV test scores of SOCrefractory versus SOC-recurrent subgroups at their symptomatic visits showed no significant differences by $\mathrm{pH}$, Nugent, or LbRC scores ( $p>0.324$, Mann-Whitney tests). Amsel scores were surprisingly better for SOC-refractory patients, mean $3.0 \pm 1.1$, versus $3.7 \pm 0.8$ for SOC-recurrent patients $(p=0.001)$. These scores do not support the hypothesis that patients were refractory because they have a more severe or dysbiotic status than the SOCrecurrent subgroup.

These data indicate that patients failing with symptomatic BV after SOC versus HDM had similar BV test scores. It was not the case that the cohort receiving HDM therapy was composed of patients with more severe cases of BV as measured by the $4 \mathrm{BV}$ diagnostic tests. It was also not the case that patients in remission from either therapy had superior BV test scores than the other therapy group, other than a marginally better mean $\mathrm{pH}$ after SOC therapy. Thus, the two therapies had similar short-term efficacies by all criteria.

\section{Long-term efficacies of SOC versus HDM}

The proportion of patients in remission versus recurrent versus refractory groups was not significantly different following SOC (23\%, 38\%, 39\%, respectively) than following HDM therapy $(21 \%, 47 \%, 32 \%$, respectively), chi-square $p=0.608$. However, among patients who recurred after treatment, HDM provided longer intervals of remission in more patients than did SOC. Median time to recurrence was 32 days after SOC (95\% CI 21-42 days) versus 42 days after $\operatorname{HDM}(95 \%$ CI 35-56 days; $p=0.027)$. Consistently, KaplanMeier analysis (Figure 2) shows significantly longer times to recurrence for approximately $25 \%$ of the patients following HDM therapy, whether early recurrences were weighted (Gehan-Breslow-Wilcoxon test, $p=0.030$ ) or not (MantelCox test, $p=0.038$; Graph Pad Prism 6.07).

Did this longer time to recurrence after HDM reflect generally healthier status while in remission from HDM than in remission from SOC? Comparisons of BV test scores between SOC-remission and HDM-remission groups, of all patients at all visits, did not show significant differences for $\mathrm{pH}$, Nugent, or Amsel scores ( $p>0.416$; Mann-Whitney tests). LbRC scores were significantly but incrementally higher for SOC-remission, mean 7.2 \pm 6.0 for SOC-remission patients versus $5.5 \pm 6.0(p=0.013)$, indicating a slightly higher mean percent Lactobacillus species after SOC treatment, not surprising since HDM patients were the subset of patients that previously failed after SOC. Similarly, comparisons of mean BV test scores per patient between SOC-remission and HDM-remission groups showed no significant differences $(p>0.471)$.

Insignificant or small magnitude differences of BV test scores between these two groups do not support the hypothesis that HDM patients in remission are in 


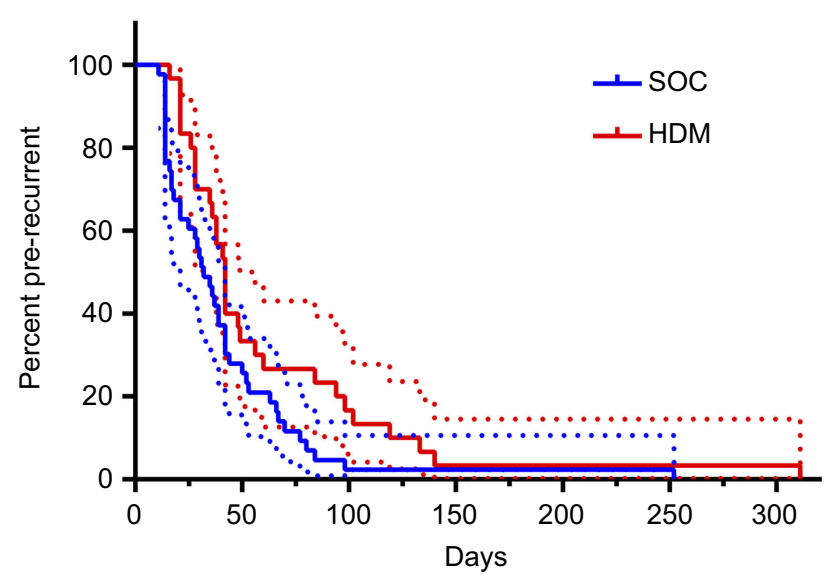

Figure 2 Kaplan-Meier analysis of recurrence rates of $\mathrm{BV}$ patients who recurred after SOC versus HDM therapy. Recurrence in this analysis was defined as Amsel 3-4 and symptoms requiring treatment. Dotted lines denote $95 \%$ confidence intervals.

Abbreviations: SOC, standard of care; BV, bacterial vaginosis; HDM, high dose vaginal metronidazole therapy

a healthier status than BV patients in remission after SOC, nor that their recurrence visits were less severe than their initial visits. Therefore, these hypotheses cannot account for the observed longer times to recurrence after HDM therapy and may require higher resolution of species by microbiomics analyses. Instead, since data indicated that mean $\mathrm{pH}$ and Amsel scores at the enrollment visit were higher and since SOC-remission patients were not included in the HDM cohort, we suggest that HDM was superior to SOC in that it achieved similar outcomes from a disadvantaged starting point.

\section{Prediction of recurrence after HDM therapy}

We previously reported that the LbRC/5 test predicted long-term outcome and Nugent predicted recurrence after SOC therapy when applied at the immediate posttreatment visit. ${ }^{30}$ In the current analysis, neither of these tests, nor Amsel and $\mathrm{pH}$, had predictive value for longterm clinical outcome when applied at the corresponding visit immediately after HDM therapy ( $p>0.272)$, nor when applied at the recurrent, symptomatic visit at which HDM therapy was initiated $(p>0.063)$. In contrast, $\mathrm{pH}$ and Amsel scores of patients at the visit just before recurrence were associated with long-term outcome (Figure 3). $\mathrm{pH}$ scores were significantly higher at the pre-HDM visit among patients who were later refractory to HDM therapy, 5.6 \pm 0.5 , compared with patients who achieved short-term remission (5.0 \pm 0.9 ) or long-term remission (4.6 \pm 0.6 ; ANOVA $p=0.039)$. $\mathrm{pH}$ below 5 at this visit had a strong NPV for at least short-term remission after therapy, 0.938 (95\% CI 0.6977 to 0.9984 ), but no significant PPV.
Likewise, Amsel scores were significantly higher at the pre-HDM visit among refractory patients $(3.2 \pm 1.5)$ than short-term remission $(1.9 \pm 2.0)$ or long-term remission patients (1.1 \pm 1.7 ; ANOVA $p=0.033)$. An Amsel score $\leq 3$ at this visit had a strong negative predictive value (NPV) for at least short-term remission after therapy, 0.889 (95\% CI 0.6529 to 0.9862 ), but no significant PPV. In the present context of prognosis, NPV is defined as the probability that a normal test result predicts subsequent remission, while PPV is the probability that an abnormal test result predicts subsequent disease. Microbiological tests, Nugent and $\mathrm{LbRC} / 5$, at this visit were not associated with clinical outcome. These data suggest that patients with prolonged albeit asymptomatic instabilities in vaginal microbial composition, reflected by $\mathrm{pH}$ and Amsel, are less effectively treated by HDM therapy.

\section{Association of menses with recurrence}

Menses in our patients is associated with time to recurrence. The mean interval between menses and recurrence after both SOC or HDM therapy is not random, instead $83 \%$ of the recurrences occurred in the first 10 days after menses. The number of recurrences occurring during weekly intervals after the last record of vaginal blood is significantly different than expected if recurrence was not associated with menses (chi sq $p=0.010$; Figure 4). The rate at which these recurrences occur is also significantly different (Kaplan-Meier analysis, Gehan-Breslow-Wilcoxon test $p=0.029$ ). Finally, 7 of 10 randomly generated recurrence patterns calculated on a daily basis are significantly different than observed (Gehan-Breslow-Wilcoxon test $p<0.05$ ); 9 of 10 such patterns were different at $p<0.07$ (data not shown).

Patients who had longer durations in and/or more frequent menses were not more likely to recur. Among tracked patients with no recurrences $(\mathrm{n}=13)$ versus one recurrence $(n=26)$ versus two recurrences $(n=10)$ during the study period, mean percentage of days reporting vaginal blood was not significantly different between the groups, $16 \%, 14 \%$, and $18 \%$, respectively (Kruskal-Wallis $p=0.958$ ).

\section{Lack of association of coitus with recurrence}

No evidence supported the hypothesis that sexual intercourse was driving recurrence in this cohort regardless of treatment regimen. There were no significant differences in distributions of patients that remained in remission versus recurred after SOC or HDM, whether coitus was with condoms or without (Table 1). Similarly, there was no correlation between remission versus recurrent groups in the number 

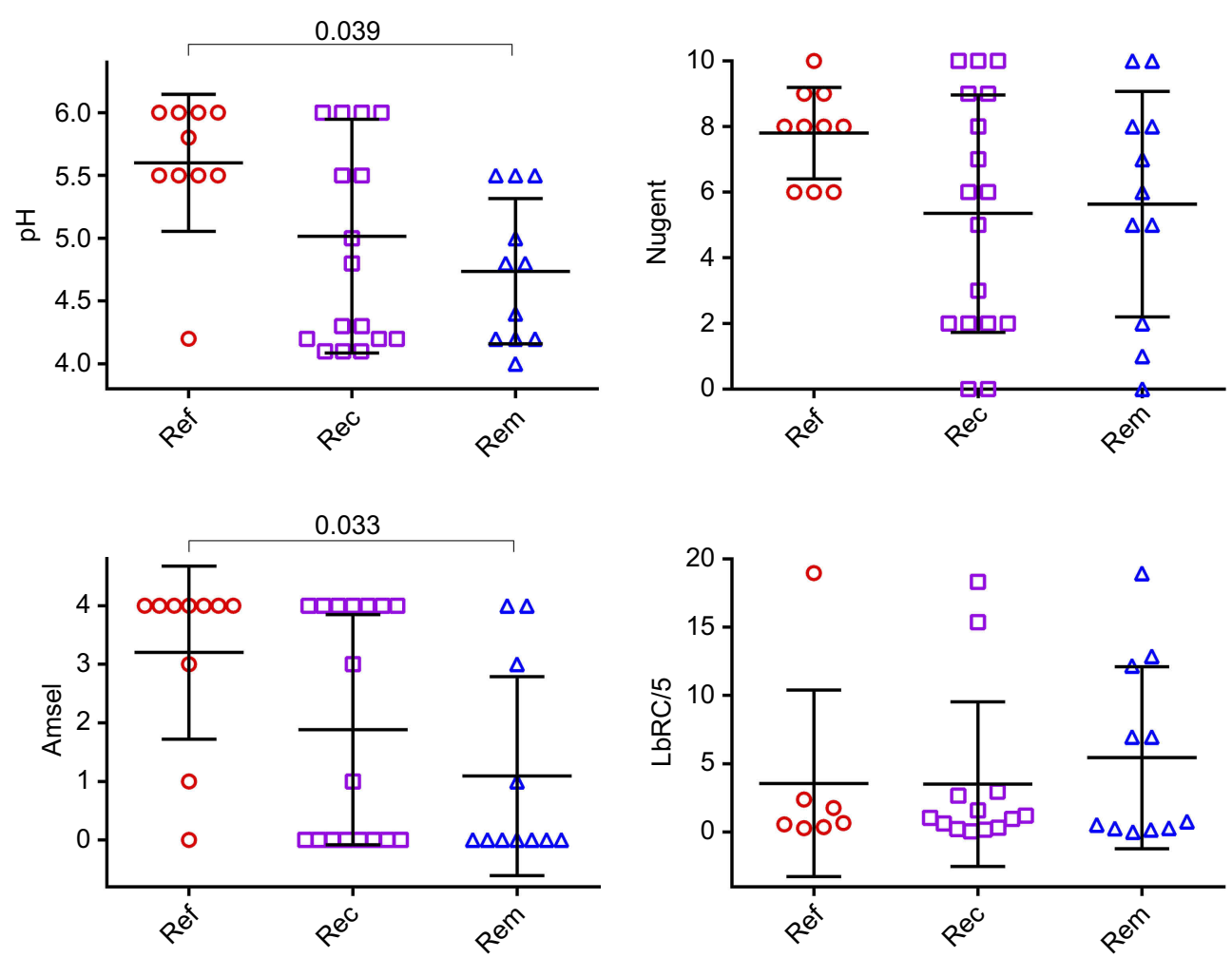

Figure 3 Association of BV diagnostic test scores at the visit preceding recurrence and HDM therapy, with clinical outcome after HDM therapy.

Notes: Ref: refractory after HDM, Rec: recurrent after HDM, Rem: remission after HDM. P-values were calculated from ANOVA; the comparisons driving low $p$-values for $\mathrm{pH}$ and Amsel were refractory versus remission groups.

Abbreviations: BV, bacterial vaginosis; HDM, high dose vaginal metronidazole therapy.

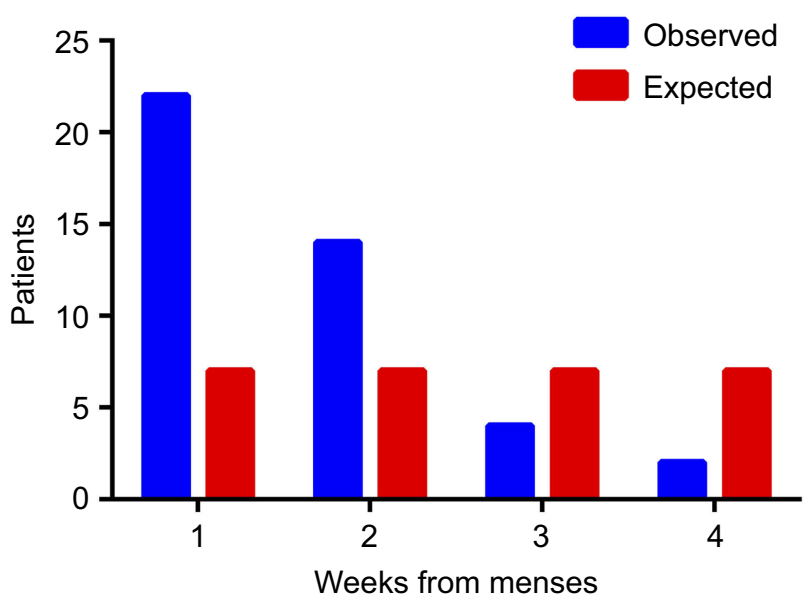

Figure 4 Time to recurrence from menses. Number of patients who recurred with symptomatic bacterial vaginosis (sBV) in the weeks following the last incidence of vaginal blood were plotted next to numbers expected if recurrence was randomly associated with menses. This number was the total number of recurrences distributed equally over 4 weeks. Observed was significantly different than expected from the null hypothesis that menses and recurrence were not associated, Chi-sq $p=0.010$.

of coitus events per patient or the percent of coitus events of total scored visits (Kruskal-Wallis tests; data not shown).

Consistently, coitus did not significantly decrease the duration of remission before recurrence after SOC or HDM, regardless of whether coitus was protected (condom) or unprotected. Days to recurrence were widely diverse in all three groups: no coitus (mean 59 days $95 \%$ CI $41-78$ days), any coitus (mean 68 days $95 \%$ CI $45-93$ days), and unprotected coitus (mean 49 days 95\% CI 29-68 days); differences between groups were not significant (Mann-Whitney $p>0.383$ ).

\section{Treatment outcome is affected by race}

The impact of race on treatment outcome is difficult to assess in this study because of the disproportionally small (6) number of scorable Caucasians compared to African Americans (68), and because of varying numbers of sequential samples between patients. As a preliminary indication of this impact, we compared distributions of mean test scores from each patient, at all visits for which the patient was in clinical remission. Table 2 indicates that the African American group had significantly higher mean $\mathrm{pH}$ and Amsel scores, and lower mean LbRC/5 scores, than the Caucasian group, while in remission from therapy. This suggests a less than ideal response to therapy while in remission among African Americans. Nugent score differences did not achieve significance, but they trended in this same direction. 
Table I Lack of association of coitus with remission versus recurrence

\begin{tabular}{|l|l|l|l|}
\hline & Remission & Recurrent & $P$-value \\
\hline Abstinent & $4(22)$ & $25(45)$ & 0.105 \\
Any coitus & $14(78)$ & $31(55)$ & \\
Coitus with condom & $5(28)$ & $13(23)$ & 0.274 \\
Coitus, no condom & $9(50)$ & $18(32)$ & 0.117 \\
\hline
\end{tabular}

Notes: Numbers (percentages) of patients in each outcome category grouped by sexual activity class. Refractory patients were not included because of their relatively short enrollment period; these patients had higher mean abstinence while in the study. Percentages were calculated based on the sums of abstinent plus any coitus. $P$-values were calculated using Fisher's exact tests, comparing each row with abstinence values.

Table 2 Impact of race on diagnostic scores

\begin{tabular}{|l|l|l|l|l|l|}
\hline $\begin{array}{l}\text { All remis- } \\
\text { sion visits }\end{array}$ & $\begin{array}{l}\text { African } \\
\text { American }\end{array}$ & SEM & Caucasian & SEM & P-value \\
\hline $\mathrm{pH}$ & 4.5 & 0.6 & 4.1 & 0.5 & 0.018 \\
Amsel & 0.57 & 0.13 & 0.06 & 0.04 & 0.0005 \\
LbRC/5 & 5.4 & 0.6 & 8.7 & 0.8 & 0.004 \\
Nugent & 3.6 & 1.3 & 3.0 & 1.3 & 0.699 \\
\hline
\end{tabular}

Notes: Mean scores for each patient (3-10 visits each) were compared between groups using unpaired $t$-tests, in some cases with Welch's correction for unequa standard deviations between groups. Remission visits were those which were not Amsel positive and did not show symptoms that warranted treatment. Remission visits included those that followed SOC or HDM therapies.

Abbreviations: SOC, standard of care; HDM, high dose vaginal metronidazole therapy; LbRC, Lactobacillus relative composition.

\section{Discussion}

The main finding of this study of RBV patients was that HDM as a follow-up therapy to SOC therapy was beneficial. Among 38 patients who recurred after or were refractory to SOC, and who remained in the study, 26 (68\%) achieved at least short-term remission from HDM follow-up therapy; 8 of these (21\%) achieved long-term remission. This beneficial effect was not as evident among patients who were refractory to SOC; 10 of 21 of these patients $(48 \%)$ were refractory a second time. We could not ethically measure the efficacy of a second, repeat regimen of SOC in patients already refractory to SOC. Unfortunately, patient attrition after receiving HDM was high $(25 \%)$ in this difficult patient population; however, it is reasonable to speculate that a disproportionally large number of these initially desperate women did not return because they achieved remission from HDM therapy. If so, HDM could be seen as more beneficial than our current estimates. HDM also extended the median interval in remission from 32 days after SOC to 42 days after HDM. While follow-up HDM therapy benefited some patients who failed SOC and increased their time to recurrence, this study found no diagnostic metric to explain its additional and extended remissions. Furthermore, this therapy is still not a major advance, since $79 \%$ of this subpopulation were not cured. This likely reflects the overall disappointing efficiency of the antimicrobial-only approach to the treatment of RBV.

Patients who were refractory to SOC were 4 times more likely to be refractory also to HDM, compared to HDM responses of patients that achieved at least short-term remission to SOC. The low-resolution BV diagnostic tests used in the present study did not detect significant differences at the initial, symptomatic visits between patients who were refractory to SOC compared to those who achieved at least shortterm remission, nor between these two groups at their recurrence visits at which they received HDM. Thus, it does not seem that the less successful outcome is due to more severe dysbiosis, as estimated, eg, by the $\mathrm{LbRC} / 5$ assay, of the refractory group compared to the remission group during symptomatic BV. This may change as the samples are analyzed by next-generation sequencing. However, other plausible explanations for this difference in outcome, which would elude convention 16S ribosomal DNA sequencing, include that the double refractory patients host metronidazole-resistant strains of $G$. vaginalis or Atopobium vaginae or other strain variants, ${ }^{6,33-36}$ that they have immune deficiencies or polymorphisms, ${ }^{37-39}$ or that they persistently engage in undocumented behaviors that increased their risk of recurrence. ${ }^{40}$ Refractory or recurrent responses may also stem from higher individual levels of polymicrobial biofilm that form on the vaginal epithelia, likely seeded by some clades of $G$. vaginalis, ${ }^{41}$ since these persist in vivo after metronidazole therapy, ${ }^{42}$ and since in vitro, vaginal bacteria in biofilms are more resistant to hydrogen peroxide and lactic acid. $^{43}$

Our cohort suffered higher recurrence rates at all intervals after SOC treatment than 7 comparable studies of less recurrent cohorts using identical or similar oral metronidazole treatments, for which recurrence was assessed at varying intervals. At 30 days, we saw 34\% recurrence compared to $10-22 \%{ }^{44,45}$ At 50 days, we saw 58\% recurrence, compared to $20 \%{ }^{1}$ At 90 days, we saw $72 \%$ recurrence, compared to $38 \%{ }^{28}$ At 180 days, we had $76 \%$ recurrence, compared to $24 \%, 28 \%$, or $32 \% .^{26,44,46}$ This higher recurrence likely results from the fact that ours is a referral clinic for recalcitrant BV patients. This implies that the benefits that we nonetheless saw from follow-up treatment with HDM may underestimate its benefit to the general population.

The use of probiotics, typically species of Lactobacillus, as a supplement to metronidazole is widely studied but controversial; some meta-analyses report increased cure rates or 
longer times to recurrence, ${ }^{47}$ while others do not support these conclusions, ${ }^{48,49}$ very few track whether the probiotic strain persists vaginally. Several individual studies show clinical benefits, using either L. crispatus, ${ }^{50}$ mixtures of Lactobacillus species ${ }^{51}$ or Lactobacillus rhamnosus BMX $54,{ }^{51}$ the latter showing benefits for at least 9 months. These studies typically are randomized control trials in which one group receives the probiotic after the 7-day metronidazole regimen. Depending on the cohort, a significant to substantial portion of those receiving probiotic would have fared well without it, which minimizes the comparative impact of the probiotic. Our previous study suggests a better assessment would be to randomize for probiotics versus not, only those patients who have $\mathrm{LbRC} / 5$ scores that predict recurrence after metronidazole therapy. ${ }^{30}$

The LbRC/5 score of samples taken immediately postSOC therapy was shown previously to be prognostic of later recurrence with high PPV (90\%) and useful NPV (74\%). ${ }^{30}$ In contrast, the present study showed that this score was not prognostic of recurrence after HDM therapy. The basis for the lack of post-HDM prognostic value is unclear and under investigation; it may be as simple as timing, ie, the interval after SOC to present prognostic information may be different than after HDM. Also, fewer patients were treated with HDM, weakening the statistical analysis. However, the visit preceding recurrence did carry prognostic information of the outcome of HDM therapy. Only Amsel scores $\leq 2$ and $\mathrm{pH}$ scores $<5$ at this pre-recurrence visit were prognostic of at least short-term remission after HDM therapy. They did not distinguish short-term remission from long-term remission. This suggests that patients whose dysbiosis before symptomatic recurrence was not protracted were more likely to achieve remission after HDM therapy, compared to patients who were dysbiotic for a month or more before symptoms arose. If validated in a larger study, this suggests that monitoring of responses of RBV patients to treatment and intervention when $\mathrm{pH}$ or Amsel scores increase, before patients become symptomatic, may improve clinical outcome.

Recurrence was associated with menses in our study in that most women who recurred did so within 10 days of menses. It is reported that menses drives declines in vaginal Lactobacillus species and/or increases in BV-associated species that are consistent with promoting recurrence. ${ }^{52-54}$ Hormonal contraception use decreases BV recurrence rates, perhaps stemming from reduced menses. ${ }^{55-58}$ This is a rational association in that the increase in $\mathrm{pH}$ and available iron from vaginal blood negate the advantages Lactobacillus species exploit before menses. We did not see associations with percent of days with vaginal blood with recurrence. Since the timing but not the prevalence of vaginal blood was associated with recurrence, we suggest that menses is a deciding factor for recurrence only at those times when vaginal dysbiosis is already at a tipping point. Further study is needed to determine if the volume or severity of menses, or other causes of vaginal blood, are contributing to recurrence.

Sexual activity, protected by condoms or not, was not associated with recurrence from SOC or HDM therapy in this study, nor did it reduce the duration of remission. This lack of association is consistent with the strong association between recurrence and the less than ideal microbial compositions revealed by the LbRC/5 assay immediately after SOC therapy, ${ }^{30}$ such an association would have been blurred if reinfection by coitus at random points thereafter was a driving force. Recurrence by relapse is also suggested by the observed association with menses.

This study was not designed to examine the role of sexual transmission in the acquisition of BV. Overwhelmingly, studies that are designed to ask this question have shown relative risks of sex with new or multiple male partners at $\sim 1.6$ and of any female partner at $\sim 2.0 .^{59-64}$ This and microbiological data ${ }^{11,65-67}$ suggest that individual females often acquire BV-associated species and incident BV via sex. Our study, in contrast, evaluates whether repeated recurrences in a highly recurrent cohort are associated with coitus or not; if not, the recurrences are likely more often due to relapse. We did not find evidence that such recurrent episodes were related to coitus (Table 1), within the limitations of our study design. Although we did not collect data on partner circumcision status, male partners in this locale are predominantly circumcised, which may reduce the risk of reinfection ${ }^{68}$ and therefore may have contributed to our observed lack of association.

Increased prevalence of BV among African Americans is well documented but poorly understood. This study showed, albeit with limited data from Caucasians, that African Americans had significantly poorer mean BV diagnostic test scores in asymptomatic remission following treatment than did Caucasian patients in remission. If this trend holds in a larger study, it suggests that African Americans recur more often than Caucasians because their microbiological response to therapy is less quantitatively or qualitatively robust. This suggests that African Americans may be especially considered for preemptive therapy after their initial treatment if post-treatment scores are not ideal. 
This pilot study has several limitations. It was not primarily aimed at comparing the overall efficacy of SOC versus HDM therapies. Such an aim will be best achieved by a large multicenter randomized, blinded, control parallel study in BV patients with more diverse demographics, including patients with sporadic and refractory BV. Instead, the present study has limited efficacy comparisons which are based upon the clinical reality in which oral metronidazole is the dominantly available and most widely prescribed agent. The high dose vaginal metronidazole is unavailable in most countries and is prescribed only in patients failing conventional therapy, hence the rationale for its use in the present study as a follow-up therapy. Moreover, in the limited comparisons performed in the present study, HDM was not given to patients with sporadic BV or to those achieving long-term remission following SOC; hence, only the failures of SOC treatment were evaluated. Other limitations include the relatively small number of patients tracked after HDM, the single geographic site of enrollment, and a disproportionally low percentage of Caucasian BV patients. This pilot study provides parameters for power analysis for the design of a larger follow-up trial.

Despite limitations, this study does suggest that HDM therapy is moderately beneficial for RBV patients following recurrence from SOC, and to a lesser extent following a refractory response to SOC. It also suggests that a larger study should investigate the merits of monitoring RBV patients, possibly only by $\mathrm{pH}$, and treating preemptively to delay a likely pending recurrence of symptomatic BV.

\section{Acknowledgments}

The authors thank the many current and former Wayne State University undergraduates and staff who contributed to patient, data management and sample scoring, including: Manell Aboutaleb, Naila Baydoun, Sarah Codreanu, Kay Dedicatoria, Manar Edriss, Tamara Jebry, Michelle Hudson, Carly Malburg, Samantha Mathews, Roshan Nizamuddin, Nasheen Nizamuddin, Andrea Prenkocevic, and Sharmi Purkayestha.

\section{Disclosure}

Drs. Sobel and Akins are co-inventors of the LbRC technology, Patent 14/782,449 to Wayne State University, Detroit, MI, USA. Dr Akins reports grants from NIAID, during the conduct of the study. The authors report no other conflicts of interest in this work.

\section{References}

1. Bradshaw CS, Morton AN, Hocking J, et al. High recurrence rates of bacterial vaginosis over the course of 12 months after oral metronidazole therapy and factors associated with recurrence. $J$ Infect Dis. 2006;193(11):1478-1486. doi:10.1086/503780

2. Hay P. Recurrent bacterial vaginosis. Curr Opin Infect Dis. 2009;22 (1):82-86. doi:10.1097/QCO.0b013e32832180c6

3. Knupp de Souza DM, Diniz CG, Filho DS, et al. Antimicrobial susceptibility and vaginolysin in Gardnerella vaginalis from healthy and bacterial vaginosis diagnosed women. J Infect Dev Ctries. 2016;10(9):913-919. doi:10.3855/jidc.7161

4. Gottschick C, Szafranski SP, Kunze B, et al. Screening of compounds against Gardnerella vaginalis biofilms. PLoS One. 2016;11(4): e0154086. doi:10.1371/journal.pone. 0154086

5. De Backer E, Verhelst R, Verstraelen H, et al. Antibiotic susceptibility of Atopobium vaginae. BMC Infect Dis. 2006;6:51. doi:10.1186/14712334-6-51

6. Ferris MJ, Masztal A, Aldridge KE, Fortenberry JD, Fidel PL Jr., Martin DH. Association of Atopobium vaginae, a recently described metronidazole resistant anaerobe, with bacterial vaginosis. $B M C$ Infect Dis. 2004;4:5. doi:10.1186/1471-2334-4-5

7. Muzny CA, Schwebke JR. Suspected heterosexual transmission of bacterial vaginosis without seminal fluid exposure. Sex Trans Dis. 2014;41(1):58-60. doi:10.1097/OLQ.0000000000000057

8. Muzny CA, Schwebke JR. Pathogenesis of bacterial vaginosis: discussion of current hypotheses. J Infect Dis. 2016;214(Suppl 1):S1S5. doi:10.1093/infdis/jiw121

9. Zabor EC, Klebanoff M, Yu K, et al. Association between periodontal disease, bacterial vaginosis, and sexual risk behaviours. $J$ Clin Periodontol. 2010;37(10):888-893. doi:10.1111/j.1600-051X.2010. 01593.x

10. Vodstrcil LA, Walker SM, Hocking JS, et al. Incident bacterial vaginosis (BV) in women who have sex with women is associated with behaviors that suggest sexual transmission of BV. Clin Infect Dis. 2015;60(7):1042-1053. doi:10.1093/cid/ciu1130

11. Zozaya M, Ferris MJ, Siren JD, et al. Bacterial communities in penile skin, male urethra, and vaginas of heterosexual couples with and without bacterial vaginosis. Microbiome. 2016;4:16. doi:10.1186/ s40168-016-0161-6

12. Genc MR, Vardhana S, Delaney ML, Witkin SS, Onderdonk AB. TNFA-308G $>$ A polymorphism influences the TNF-alpha response to altered vaginal flora. Eur J Obstet Gynecol Reprod Biol. 2007;134 (2):188-191. doi:10.1016/j.ejogrb.2006.10.018

13. Peixoto F, Camargos A, Duarte G, Linhares I, Bahamondes L, Petracco A. Efficacy and tolerance of metronidazole and miconazole nitrate in treatment of vaginitis. Intl J Gynecol Obstet. 2008;102 (3):287-292. doi:10.1016/j.ijgo.2008.04.014

14. Sanchez S, Garcia PJ, Thomas KK, Catlin M, Holmes KK. Intravaginal metronidazole gel versus metronidazole plus nystatin ovules for bacterial vaginosis: a randomized controlled trial. $\mathrm{Am}$ $J$ Obstet Gynecol. 2004;191(6):1898-1906. doi:10.1016/j. ajog.2004.06.089

15. Ozyurt E, Toykuliyeva MB, Danilyans IL, Morton O, Baktir G. Efficacy of 7-day treatment with metronidazole+miconazole (Neo-Penotran) - a triple-active pessary for the treatment of single and mixed vaginal infections. Intl $J$ Gynecol Obstet. 2001;74 (1):35-43. doi:10.1016/S0020-7292(01)00388-5

16. Aguin T, Akins RA, Sobel JD. High-dose vaginal maintenance metronidazole for recurrent bacterial vaginosis: a pilot study. Sex Trans Dis. 2014;41(5):290-291. doi:10.1097/OLQ.000000000000 0123 
17. Aguin TJ, Akins RA, Sobel JD. High-dose vaginal metronidazole for recurrent bacterial vaginosis-a pilot study. J Lower Genital Tract Dis. 2014;18(2):156-61. doi:10.1097/LGT.0b013e31829a5558

18. Algburi A, Volski A, Chikindas ML. Natural antimicrobials subtilosin and lauramide arginine ethyl ester synergize with conventional antibiotics clindamycin and metronidazole against biofilms of Gardnerella vaginalis but not against biofilms of healthy vaginal lactobacilli. Pathogens Dis. 2015;73(5)

19. Cavera VL, Volski A, Chikindas ML. The natural antimicrobial subtilosin a synergizes with Lauramide Arginine Ethyl Ester (LAE), epsilon-Poly-L-lysine (Polylysine), clindamycin phosphate and metronidazole, against the vaginal pathogen Gardnerella vaginalis. Probiotics Antimicrob Prot. 2015;7(2):164-171. doi:10.1007/s12602014-9183-1

20. Masoudi M, Miraj S, Rafieian-Kopaei M. Comparison of the effects of Myrtus Communis L, Berberis Vulgaris and metronidazole vaginal gel alone for the treatment of bacterial vaginosis. J Clin Diag Res. 2016;10(3):Qc04-Qc07.

21. Reichman O, Akins R, Sobel JD. Boric acid addition to suppressive antimicrobial therapy for recurrent bacterial vaginosis. Sex Trans Dis. 2009;36(11):732-734. doi:10.1097/OLQ.0b013e3181b08456

22. Petersen EE, Genet M, Caserini M, Palmieri R. Efficacy of vitamin $\mathrm{C}$ vaginal tablets in the treatment of bacterial vaginosis: a randomised, double blind, placebo controlled clinical trial. Arzneimittelforschung. 2011;61(4):260-265. doi:10.1055/s-0031-1296197

23. Verstraelen H, Vervaet C, Remon JP. Rationale and safety assessment of a novel intravaginal drug-delivery system with sustained DL-lactic acid release, intended for long-term protection of the vaginal microbiome. PLoS One. 2016;11(4):e0153441. doi:10.1371/journal. pone. 0153441

24. Otsuki K, Imai N. Effects of lactoferrin in 6 patients with refractory bacterial vaginosis. Biochem Cell Biol. 2017;95(1):31-33. doi:10.1139/bcb-2016-0051

25. Russo R, Edu A, De Seta F. Study on the effects of an oral lactobacilli and lactoferrin complex in women with intermediate vaginal microbiota. Arch Gynecol Obstet. 2018;298(1):139-145. doi:10.1007/s00404-018-4771-z

26. Russo R, Karadja E, De Seta F. Evidence-based mixture containing Lactobacillus strains and lactoferrin to prevent recurrent bacterial vaginosis: a double blind, placebo controlled, randomised clinical trial. Benef Microbes. 2019;10(1):19-26. doi:10.3920/BM2018.0075

27. Collins SL, McMillan A, Seney S, et al. Promising prebiotic candidate established by evaluation of lactitol, lactulose, raffinose, and oligofructose for maintenance of a lactobacillus-dominated vaginal microbiota. Appl Environ Microbiol. 2018;84:5. doi:10.1046/j.13652672.1997.00313.x

28. Hakimi S, Farhan F, Farshbaf-Khalili A, et al. The effect of prebiotic vaginal gel with adjuvant oral metronidazole tablets on treatment and recurrence of bacterial vaginosis: a triple-blind randomized controlled study. Arch Gynecol Obstet. 2018;297(1):109-116. doi:10.1007/s00404-017-4555-x

29. Balkus JE, Srinivasan S, Anzala O, et al. Impact of periodic presumptive treatment for bacterial vaginosis on the vaginal microbiome among women participating in the preventing vaginal infections trial. J Infect Dis. 2017;215(5):723-731. doi:10.1093/infdis/jiw622

30. Sobel JD, Kaur N, Woznicki NA, et al. Prognostic indicators of recurrence of bacterial vaginosis. J Clin Microbiol. 2019. doi:10.1128/JCM.00227-19

31. Amsel R, Totten PA, Spiegel CA, Chen KC, Eschenbach D, Holmes KK. Nonspecific vaginitis. Diagnostic criteria and microbial and epidemiologic associations. Am J Med. 1983;74(1):14-22.

32. Bossuyt PM, Cohen JF, Gatsonis CA, Korevaar DA, group S. STARD 2015: updated reporting guidelines for all diagnostic accuracy studies. Ann Transl Med. 2016;4(4):85. doi:10.21037/ atm.2016.04.05
33. Nagaraja P. Antibiotic resistance of Gardnerella vaginalis in recurrent bacterial vaginosis. Indian $J$ Med Microbiol. 2008;26 (2):155-157.

34. Schuyler JA, Mordechai E, Adelson ME, Sobel JD, Gygax SE, Hilbert DW. Identification of intrinsically metronidazole-resistant clades of Gardnerella vaginalis. Diagn Microbiol Infect Dis. 2016;84(1):1-3. doi:10.1016/j.diagmicrobio.2015.10.006

35. Deng ZL, Gottschick C, Bhuju S, Masur C, Abels C, Wagner-Dobler I. Metatranscriptome analysis of the vaginal microbiota reveals potential mechanisms for protection against metronidazole in bacterial vaginosis. mSphere. 2018;3:3. doi:10.1128/mSphereDirect.0026218

36. Austin MN, Meyn LA, Hillier SL. Susceptibility of vaginal bacteria to metronidazole and tinidazole. Anaerobe. 2006;12(5-6):227-230. doi:10.1016/j.anaerobe.2006.06.002

37. Demirbilek M, Can F, Gulec AT, Kuscu E, Kayhan Z, Haberal M. Incidence of bacterial vaginosis in renal transplant recipients. Transplant Proc. 2003;35(7):2696-2697.

38. Genc MR, Onderdonk A. Endogenous bacterial flora in pregnant women and the influence of maternal genetic variation. BJOG. 2011;118(2):154-163. doi:10.1111/j.1471-0528.2010.02772.x

39. Mitchell C, Marrazzo J. Bacterial vaginosis and the cervicovaginal immune response. Am J Reprod Immunol. 2014;71(6):555-563. doi:10.1111/aji.12264

40. Cherpes TL, Hillier SL, Meyn LA, Busch JL, Krohn MA. A delicate balance: risk factors for acquisition of bacterial vaginosis include sexual activity, absence of hydrogen peroxide-producing lactobacilli, black race, and positive herpes simplex virus type 2 serology. Sex Trans Dis. 2008;35(1):78-83. doi:10.1097/OLQ.0b013e318156a5d0

41. Swidsinski A, Mendling W, Loening-Baucke V, et al. Adherent biofilms in bacterial vaginosis. Obstet Gynecol. 2005;106(5 Pt 1):1013-1023. doi:10.1097/01.AOG.0000183594.45524.d2

42. Swidsinski A, Mendling W, Loening-Baucke V, et al. An adherent Gardnerella vaginalis biofilm persists on the vaginal epithelium after standard therapy with oral metronidazole. Am J Obstet Gynecol. 2008;198(1):97.e91-97.e96. doi:10.1016/j.ajog.2007.06.039

43. Patterson JL, Girerd PH, Karjane NW, Jefferson KK. Effect of biofilm phenotype on resistance of Gardnerella vaginalis to hydrogen peroxide and lactic acid. Am J Obstet Gynecol. 2007;197(2):170. e171-170.e177. doi:10.1016/j.ajog.2007.02.027

44. Bradshaw CS, Pirotta M, De Guingand D, et al. Efficacy of oral metronidazole with vaginal clindamycin or vaginal probiotic for bacterial vaginosis: randomised placebo-controlled double-blind trial. PLoS One. 2012;7(4):e34540. doi:10.1371/journal. pone. 0034540

45. Lugo-Miro VI, Green M, Mazur L. Comparison of different metronidazole therapeutic regimens for bacterial vaginosis. A meta-analysis. JAMA. 1992;268(1):92-95.

46. Krasnopolsky VN, Prilepskaya VN, Polatti F, et al. Efficacy of vitamin $\mathrm{C}$ vaginal tablets as prophylaxis for recurrent bacterial vaginosis: a randomised, double-blind, placebo-controlled clinical trial. J Clin Med Res. 2013;5(4):309-315. doi:10.4021/jocmr1489w

47. Huang H, Song L, Zhao W. Effects of probiotics for the treatment of bacterial vaginosis in adult women: a meta-analysis of randomized clinical trials. Arch Gynecol Obstet. 2014;289(6):1225-1234. doi:10.1007/s00404-013-3117-0

48. Buggio L, Somigliana E, Borghi A, Vercellini P. Probiotics and vaginal microecology: fact or fancy? BMC Women's Health. 2019;19(1):25. doi:10.1186/s12905-019-0723-4

49. Tan H, Fu Y, Yang C, Ma J. Effects of metronidazole combined probiotics over metronidazole alone for the treatment of bacterial vaginosis: a meta-analysis of randomized clinical trials. Arch Gynecol Obstet. 2017;295(6):1331-1339. doi:10.1007/s00404-0174366-0 
50. Bohbot JM, Darai E, Bretelle F, Brami G, Daniel C, Cardot JM. Efficacy and safety of vaginally administered lyophilized Lactobacillus crispatus IP 174178 in the prevention of bacterial vaginosis recurrence. J Gynecol Obstet Human Reprod. 2018;47 (2):81-86. doi:10.1016/j.jogoh.2017.11.005

51. Laue C, Papazova E, Liesegang A, et al. Effect of a yoghurt drink containing Lactobacillus strains on bacterial vaginosis in women - a double-blind, randomised, controlled clinical pilot trial. Benef Microbes. 2018;9(1):35-50. doi:10.3920/BM2017.0018

52. Lambert JA, John S, Sobel JD, Akins RA. Longitudinal analysis of vaginal microbiome dynamics in women with recurrent bacterial vaginosis: recognition of the conversion process. PLoS One. 2013;8 (12):e82599. doi:10.1371/journal.pone.0082599

53. Srinivasan S, Liu C, Mitchell CM, et al. Temporal variability of human vaginal bacteria and relationship with bacterial vaginosis. PLoS One. 2010;5(4):e10197. doi:10.1371/journal.pone.0010197

54. Fredricks DN. Molecular methods to describe the spectrum and dynamics of the vaginal microbiota. Anaerobe. 2011;17(4):191-195. doi:10.1016/j.anaerobe.2011.01.001

55. Bradshaw CS, Vodstrcil LA, Hocking JS, et al. Recurrence of bacterial vaginosis is significantly associated with posttreatment sexual activities and hormonal contraceptive use. Clin Infect Dis. 2013;56 (6):777-786. doi:10.1093/cid/cis1030

56. Riggs M, Klebanoff M, Nansel T, Zhang J, Schwebke J, Andrews W. Longitudinal association between hormonal contraceptives and bacterial vaginosis in women of reproductive age. Sex Transm Dis. 2007;34(12):954-959.

57. van de Wijgert JH, Verwijs MC, Turner AN, Morrison CS. Hormonal contraception decreases bacterial vaginosis but oral contraception may increase candidiasis: implications for HIV transmission. AIDS. 2013;27(13):2141-2153. doi:10.1097/QAD.0b013e32836290b6

58. Vodstrcil LA, Hocking JS, Law M, et al. Hormonal contraception is associated with a reduced risk of bacterial vaginosis: a systematic review and meta-analysis. PLoS One. 2013;8(9):e73055. doi:10.1371/ journal.pone.0073055

59. Fethers KA, Fairley CK, Hocking JS, Gurrin LC, Bradshaw CS. Sexual risk factors and bacterial vaginosis: a systematic review and meta-analysis. Clin Infect Dis. 2008;47(11):1426-1435. doi:10.1086/ 592974
60. Muzny CA, Lensing SY, Aaron KJ, Schwebke JR. Incubation period and risk factors support sexual transmission of bacterial vaginosis in women who have sex with women. Sex Trans Infect. 2019. doi:10.1136/sextrans-2018-053824

61. Ignacio MAO, Andrade J, Freitas APF, Pinto G, Silva MGD, Duarte MTC. Prevalence of bacterial vaginosis and factors associated among women who have sex with women. Rev Lat Am Enfermagem. 2018;26:e3077. doi:10.1590/1518-8345.2491.3077

62. Norris Turner A, Carr Reese P, Snead MC, et al. Recent biomarker-confirmed unprotected vaginal sex, but not self-reported unprotected sex, is associated with recurrent bacterial vaginosis. Sex Trans Dis. 2016;43(3):172-176. doi:10.1097/OLQ.0000000000000414

63. Kenyon CR, Buyze J, Klebanoff M, Brotman RM. Association between bacterial vaginosis and partner concurrency: a longitudinal study. Sex Trans Infect. 2018;94(1):75-77. doi:10.1136/sextrans2016-052652

64. Bradshaw CS, Walker J, Fairley CK, et al. Prevalent and incident bacterial vaginosis are associated with sexual and contraceptive behaviours in young Australian women. PLoS One. 2013;8(3): e57688. doi:10.1371/journal.pone.0057688

65. Elsner P, Hartmann AA. Gardnerella vaginalis in the male upper genital tract: a possible source of reinfection of the female partner Sex Trans Dis. 1987;14(2):122-123. doi:10.1097/00007435198704000-00015

66. Gottschick C, Deng ZL, Vital M, et al. The urinary microbiota of men and women and its changes in women during bacterial vaginosis and antibiotic treatment. Microbiome. 2017;5(1):99. doi:10.1186/s40168017-0305-3

67. Liu CM, Hungate BA, Tobian AA, et al. Penile microbiota and female partner bacterial vaginosis in Rakai, Uganda. mBio. 2015;6 (3):e00589. doi:10.1128/mBio.00589-15

68. Price LB, Liu CM, Johnson KE, et al. The effects of circumcision on the penis microbiome. PLoS One. 2010;5(1):e8422. doi:10.1371/journal.pone.0008422
Infection and Drug Resistance

\section{Publish your work in this journal}

Infection and Drug Resistance is an international, peer-reviewed openaccess journal that focuses on the optimal treatment of infection (bacterial, fungal and viral) and the development and institution of preventive strategies to minimize the development and spread of resistance. The journal is specifically concerned with the epidemiology of antibiotic resistance and the mechanisms of resistance development and diffusion in both hospitals and the community. The manuscript management system is completely online and includes a very quick and fair peerreview system, which is all easy to use. Visit http://www.dovepress.com/ testimonials.php to read real quotes from published authors. 